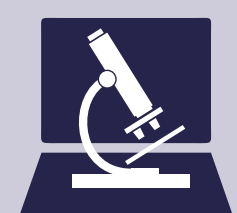

PathologyOutlines.com

\section{What's new in gynecologic pathology 2021: ovary and fallopian tube}

\author{
Gulisa Turashvilii and Ricardo Lastra ${ }^{2}$
}

'Department of Pathology and Laboratory Medicine, Sinai Health System and University of Toronto, Toronto, ON, Canada 2Department of Pathology, University of Chicago Medical Center, Chicago, IL, USA
Received: July 26, 2021 Accepted: July 28, 2021

Corresponding Author: Gulisa Turashvili, MD, PhD Department of Pathology and Laboratory Medicine Sinai Health System and University of Toronto

Toronto, Ontario, Canada

E-mail: Gulisa.Turashvili@sinaihealth.ca

ORCID

Gulisa Turashvili

https://orcid.org/0000-0001-6125-5865

Ricardo Lastra

https://orcid.org/0000-0003-0691-5685

This article has been published jointly, with consent, in both Journal of Pathology and Translational Medicine and PathologyOutlines.com.

\section{Abstract}

The 5th edition of the World Health Organization (WHO) Classification of Female Genital Tumors was published in 2020. Although the classification of ovarian and fallopian tube neoplasms is largely unchanged from the prior (4th) edition, this newsletter compiles the most important refinements in these organ sites, including serous and non-serous epithelial tumors, and sex cord-stromal tumors.

\section{SEROUS NEOPLASMS}

- The 4th edition divided serous carcinoma into low grade (LGSC) and high grade (HGSC) variants.

- LGSC and HGSC are best considered two fundamentally different tumors based on their distinct biology, rather than variants of the same neoplasm.

- Nearly all LGSCs arise in a background of ovarian benign or borderline serous tumors.

- Most HGSCs are believed to arise from a precursor lesion, STIC (serous tubal intraepithelial carcinoma), in the tubal fimbriae.

- New criteria for primary site assignment in
HGSC include:

- Fallopian tube: STIC present, or mucosal

HGSC present, or part or all of the fallopian tube is inseparable from tubo-ovarian mass.

- Ovary: both fallopian tubes are separate from ovarian mass, and no STIC or mucosal HGSC present in either fallopian tube.

- Tubo-ovarian: fallopian tubes and ovaries are unavailable for complete examination, and pathologic findings are consistent with extrauterine HGSC.

- Peritoneal (exceedingly rare): both fallopian tubes and ovaries are fully examined using a SEE-FIM (Sectioning and Extensively Examining the FIMbriated end) protocol, and no gross or microscopic evidence of STIC or HGSC present in either fallopian tube or ovary.

- These criteria classify approximately $80 \%$ of HGSCs as primary tubal.

- Serous borderline tumor is the sole recommended term:

- Obsolete terminology no longer recommended includes atypical proliferative serous tumor, serous tumor of low malignant potential, semimalignant serous tumor and non-invasive LGSC / micropapillary serous borderline tumor (the latter no longer considered definitionally synonymous with noninvasive LGSC).

\section{NON-SEROUS EPITHELIAL TUMORS}

- The following terminology is no longer recommended:

- Atypical proliferative tumor or tumor of low malignant potential for all epithelial tumors (mucinous, endometrioid, seromucinous, clear cell, Brenner).

\section{SEROMUCINOUS CARCINOMA}

- Previously defined as a carcinoma composed predominantly of serous and endocervical-type mucinous epithelium, often with foci showing clear cells, endometrioid or squamous differentiation.

- Now considered a subtype of endometrioid adenocarcinoma with mucinous differentiation (Fig. 1).

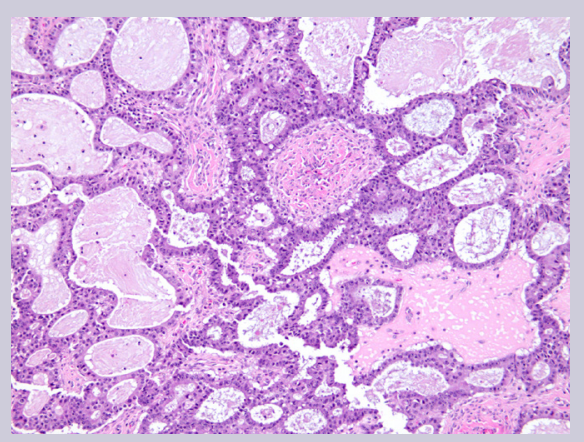

Fig. 1. Endometrioid adenocarcinoma with mucinous differentiation.

\section{NEW VARIANTS OF EPITHELIAL TUMORS}

- Mesonephric-like adenocarcinoma:

- Composed of multiple architectural patterns (tubular, glandular/pseudoendometrioid, ductal, papillary, solid), intraluminal eosinophilic colloid-like material, dense or vesicular chromatin, inconspicuous nucleoli and nuclear crowding, and lacking squamous or mucinous differentiation.

- Positive for GATA3, TTF1, CD10 (luminal) and PAX8, and negative for hormone receptors and WT1, with wild-type p53 expression.

- Usually unilateral and diagnosed at stage I in postmenopausal women. 
- May arise from paraovarian mesonephric remnants or Müllerian carcinomas displaying secondary mesonephric transdifferentiation.

- May be associated with endometriosis, cystadenomas, adenofibromas, borderline tumors and LGSC.

- The most common molecular alterations include KRAS mutations, $1 \mathrm{p}$ loss and $1 \mathrm{q}$ gain, while NRAS or PIK3CA mutations are rare.

- Tumors with coexisting serous neoplasms show shared molecular alterations (KRAS or NRAS mutations).

- Clinical outcome is unknown due to rarity.

- Dedifferentiated carcinoma:

- A biphasic tumor composed of an undifferentiated carcinoma (sheet-like growth of monotonous, discohesive, round, rhabdoid to spindle cells with brisk mitoses, often necrosis and abundant tumor-infiltrating lymphocytes) and a differentiated (usually low grade endometrioid adenocarcinoma, rarely serous carcinoma) component, often with abrupt interface in between (Fig. 2). - Undifferentiated areas are focally positive for EMA, pan-keratin and CK18, focally positive to negative for PAX8, and negative for hormone receptors and E-cadherin, with common loss of SMARCA4 (BRG1), SMARCA2 (BRM), SMARCB1 (INI1) or ARID1A, DNA mismatch repair deficiency in onethird of cases and typically wild-type p53 expression.

- Usually diagnosed at advanced stages, with pelvic and para-aortic lymph node involvement and poor prognosis.

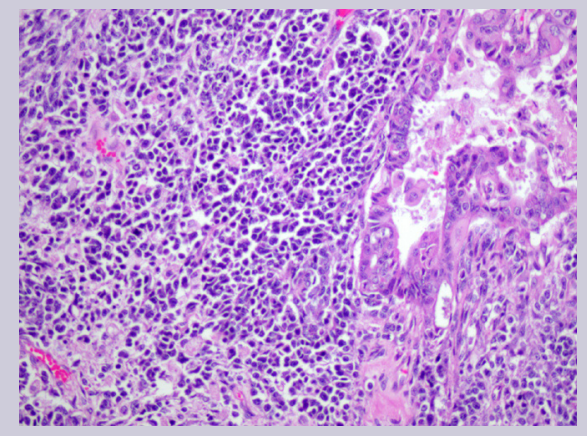

Fig. 2. Dedifferentiated carcinoma composed of undifferentiated carcinoma and low-grade endometrioid adenocarcinoma.

- Carcinosarcoma:

- A biphasic neoplasm composed of high grade carcinomatous and sarcomatous elements.

- Now considered a variant of carcinoma rather than a true mixed epithelial-mesenchymal tumor.

- Mixed carcinoma:

- True mixed carcinomas are uncommon.

- Should only be diagnosed when at least two tumor types are clearly recognizable on hematoxylin-eosin-stained sections, with distinct morphologic and preferably immunopheno- typic differences.

Each histotype with their percentages should be reported (no minimum percentage requirement).

Endometriosis-associated histotypes are most common, e.g. endometrioid and clear cell carcinomas (Fig. 3).

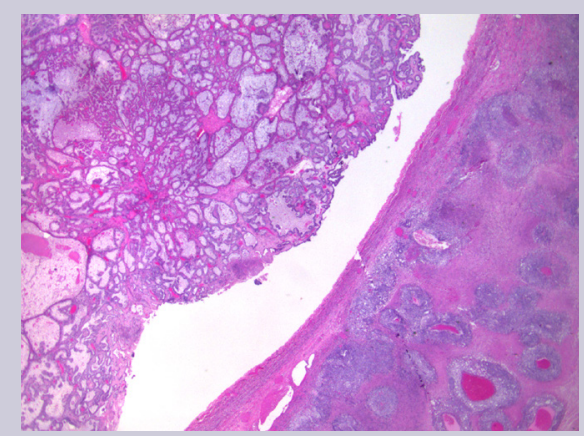

Fig. 3. Mixed carcinoma composed of clear cell carcinoma and endometrioid adenocarcinoma.

\section{ANCILLARY TESTING}

- Aberrant p53 expression refers to three immunostaining patterns associated with TP53 mutation:

- Overexpression (strong nuclear expression in $>80 \%$ of tumor cells).

- Complete absence of nuclear staining (with satisfactory controls).

- Unequivocal cytoplasmic expression.

\section{SEX CORD-STROMAL TUMORS}

- Most (>90\%) adult-type granulosa cell tumors (GCTs) exhibit somatic FOXL2 mutations.

- Sertoli-Leydig cell tumors (SLCTs) may harbor DICER 1 or FOXL2 mutations and are now classified into three molecular subtypes:

- DICER1-mutant tumors show somatic $(\sim 50 \%)$ or germline $(69 \%)$ hotspot mutations in the RNase IIIb domain of DICER1, an endoribonuclease involved in microRNA processing and gene expression regulation.

- Occur in younger patients and induce androgenic symptoms.

- Moderately or poorly differentiated with retiform or heterologous elements (the latter two predict DICER1 mutations) (Fig. 4).

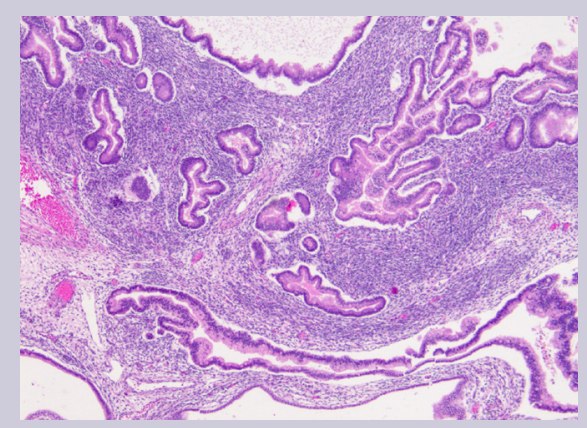

Fig. 4. Moderately differentiated Sertoli-Leydig cell tumor with heterologous elements (intestinal-type glands) and somatic DICER1 mutation.
- F0XL2-mutant tumors show c. $402 \mathrm{C}>\mathrm{G}$ (p.Cys134Trp) mutations that upregulate CYP19A1 encoding aromatase.

- Occur in postmenopausal patients and induce estrogenic symptoms.

- Moderately or poorly differentiated lacking retiform or heterologous elements.

- Reported in 0-22\% of cases.

- FOXL2 and DICER1 mutations are mutually exclusive.

- DICER1-FOXL2 wild-type tumors:

- Occur in patients with intermediate age.

- Well-differentiated lacking retiform or heterologous elements.

- Microcystic stromal tumors exhibit CTNNB1 or, less frequently, APC mutations and may represent an extracolonic manifestation of familial adenomatous polyposis.

- Small cell carcinomas of hypercalcemic type exhibit deleterious germline or somatic mutations in SMARCA4, part of the SWI/SNF complex, resulting in loss of SMARCA4 protein expression.

- Gynandroblastoma has been reintroduced (Fig. 5). - Defined as a sex cord-stromal tumor with an admixure of female (adult-type or juvenile GCT) and male (Sertoli cell tumor or SLCT) elements.

- Most commonly composed of a predominant SLCT component and a smaller component of juvenile GCT, both expressing sex cordstromal markers, sometimes with shared DICER1 mutations.

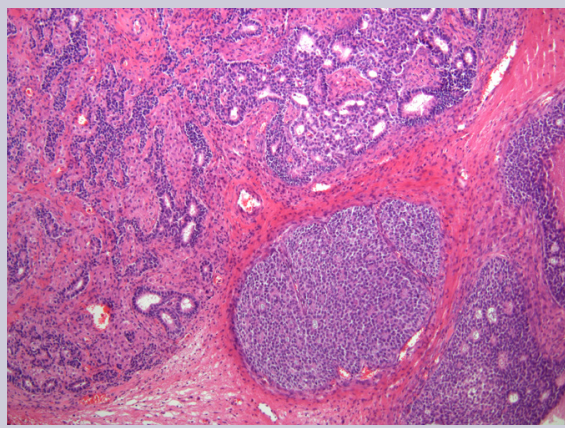

Fig. 5. Gynandroblastoma composed of SertoliLeydig cell tumor and adult-type granulosa cell tumor.

\section{Meet the Authors}

Dr. Turashvili has been part of the Pathology Outlines editorial board since 2020. She is originally from Georgia and pursued her pathology training in Canada and USA. She is currently affiliated with Mount Sinai Hospital and the University of Toronto as a gynecologic and breast pathologist and Assistant Professor.

Dr. Lastra has been part of the Pathology Outlines editorial board since 2020. He is currently an Associate Professor of pathology at the University of Chicago Medical Center, where he also serves as the gynecologic pathology fellowship program director. 